\title{
Anti-PD-1 combined sorafenib versus anti- PD-1 alone in the treatment of advanced hepatocellular cell carcinoma: a propensity score-matching study
}

San-Chi Chen 1,2,3, Yi-Hsiang Huang ${ }^{1,2,4}$, Ming-Huang Chen ${ }^{1,2,3}$, Yi-Ping Hung ${ }^{1,2,3}$, Rheun-Chuan Lee ${ }^{5}$, Yu-Yun Shao $6,7,8$ and Yee Chao $1,2,3^{*}$

\begin{abstract}
Background: Vascular endothelial growth factor (VEGF) plays a role in the tumor microenvironment. Sorafenib, which inhibits the VEGF pathway, has an immune-modulation function but lacks substantial clinical data. This study aims to explore the efficacy of anti-PD-1 combined sorafenib in advanced hepatocellular carcinoma (HCC).

Methods: HCC patients who underwent anti-PD-1 treatment at Taipei Veterans General Hospital (Taipei, Taiwan) between January 2016 and February 2019 were reviewed. The efficacy was compared between groups after propensity-score matching.

Results: There were 173 HCC patients receiving anti-PD-1. After excluding unsuitable cases, 140 patients were analyzed, of which 58 received combination therapy and 82 received anti-PD-1 alone. The combination therapy had a trend of higher CR rate ( $8.6 \%$ vs. $4.9 \%$, ns.), ORR (22.4\% vs. $19.5 \%$, ns.) and significantly higher DCR (69.0\% vs. $37.8 \%$, $p<0.05)$ comparing to anti-PD-1 alone. After matching, combination group achieved longer progression-free survival (3.87 vs. 2.43 months, $p<0.05$ ) and overall survival (not reached vs. 7.17 months, $p<0.05$ ) than anti-PD-1 alone, without higher grade $3 / 4 \mathrm{AE}(10.3 \%$ vs. $7.1 \%, p=0.73)$. The tumor response varied among different metastatic sites, with high responses in adrenal glands, peritoneum and lungs. The more AFP declined $(>10,>50$ and $>66 \%)$, the higher the ORR (70, 80 and 92\%) and CR rates (30, 35 and 58\%) were achieved at day 28.
\end{abstract}

Conclusions: This is the first study to demonstrate the combination of anti-PD-1 and sorafenib had better efficacy and survival benefit. A prospective randomized study is needed to confirm this finding.

Keywords: Hepatocellular carcinoma (HCC), Anti-PD-1, Nivolumab, Pembrolizumab, Sorafenib, Multiple-kinase inhibitor, Vascular endothelial growth factor (VEGF), Combination therapy, Propensity score matching (PSM)

*Correspondence: ychao@vghtpe.gov.tw

${ }^{3}$ Division of Medical Oncology, Center for Immuno-oncology,

Department of Oncology, Taipei Veterans General Hospital, No. 201, Sec.

2, Shipai Road, Taiwan 11217 Taipei, Taiwan

Full list of author information is available at the end of the article

\section{Background}

HCC is the second most common cause of cancerrelated death in Taiwan [1] and ranks fourth worldwide [2]. In advanced HCC, the prognosis is poor and treatment options are limited. In recent years, anti-PD-1 has become the standard treatment for many types of cancer, including HCC. However, nivolumab failed to prove its survival benefit over sorafenib in 1st line treatment [3]. 
In the absence of biomarker, to combine anti-PD-1 and other drugs becomes a feasible treatment option. The major potential drugs include anti-vascular endothelial growth factor monoclonal-antibody (anti-VEGF mAb), anti-CTLA-4 and multiple-kinase inhibitors (MKI). Among these, anti-VEGF mAb is the most successful.

Recently, vascular endothelial growth factor (VEGF) was found to play a role in the tumor microenvironment. The blockade of the VEGF pathway increases the infiltration of effector immune cells via normalization of abnormal tumor vasculature [4]. In a phase 1b study, GO30140, the addition of bevacizumab to atezolizumab demonstrated longer PFS than atezolizumab alone, which made this combination a promising treatment option for HCC [5]. Thereafter, atezolizumab combined bevacizumab resulted in better PFS and OS outcomes than sorafenib in Imbrave150 [6]. Based on these data, this regimen become the first recommended combination therapy in the first line setting of advanced HCC.

Sorafenib, a multiple kinase inhibitor that inhibits VEGF pathway, has been the first-choice drug recommendation in advanced HCC for a decade [7]. The inhibition of VEGF pathway by sorafenib not only enhances functions of effector $\mathrm{T}$ cells in tumor microenvironment [8], but also decreases suppressive immune cells [8-10]. Besides, sorafenib-treated HCC tissue significantly increased PD-L1 expression in immune cells [11]. When combined with anti-PD-1, sorafenib inhibited tumor growth by inducing effective natural killer cells [12] These data suggested that sorafenib may be a potential candidate to combine with anti-PD-1.

There was only one case report showing that the combination of anti-PD-1 and sorafenib achieved complete response with advanced HCC [13]. However, there is no study providing survival benefit with the combination of multiple-kinase inhibitor and anti-PD-1. The aim of this study is to explore the clinical benefit of the addition of sorafenib to anti-PD-1 comparing with anti-PD-1 alone.

\section{Methods}

\section{Patients and study design}

Between January 2016 and February 2019, 173 HCC patients who underwent anti-PD-1 treatment with or without sorafenib at Taipei Veterans General Hospital (Taipei, Taiwan) were retrospectively reviewed. Patients with Child-Pugh Score C or those without efficacy assessment were excluded, therefore, 140 patients were enrolled for this study. Information regarding patient characteristics, including patient age, sex, history of viral hepatitis, liver function, tumor markers, tumor stage, and tumor treatment history was collected and analyzed. The diagnosis of $\mathrm{HCC}$ was confirmed histologically or clinically based on the American Association for the Study of Liver Diseases criteria.

Liver function was established by Child-Pugh score and ALBI grade. Cancer staging used the Barcelona clinic liver cancer (BCLC) staging system. The up-to-11 criteria combined the number of tumors and size of the largest one, with the sum being no more than 11 was applied for tumor burden. This study has been approved by the institutional review board of Taipei Veterans General Hospital, which is the appropriate regulatory agency to review research on both adults and children. (VGHTPE-IRB: 2017-10-005 BC). This work was supported by grants from the Ministry of Health and Welfare and the Center of Excellence for Cancer Research (MOHW110-TDUB-211-144,019) and Taipei Veterans General Hospital (V107B-036 to S-CC).

\section{Outcome assessment}

Treatment responses were assessed by Computed Tomography scan or Magnetic Resonance Imaging every $2-3$ months in accordance with RECIST and mRECIST criteria (19). Immune-related adverse events were graded according to the National Cancer Institute Common Terminology Criteria for Adverse Events version 4.0. Progression-free survival (PFS) was defined as the time period from the beginning of treatment until disease progression or death, time to response (ToR) from the beginning of treatment until documentation of tumor response, duration of response (DoR) from documentation of tumor response to disease progression, and overall survival (OS) from the beginning of treatment to death.

\section{Statistical analysis}

To reduce confounding, propensity-score was used to match patients treated with anti-PD-1 plus sorafenib to those treated with anti-PD-1 alone. Variables including age, sex, etiology, cirrhosis, Child-Pugh score, portal vein thrombosis (PVT), metastasis, AFP level and sorafenib experienced were used for matching. Inverse probability of treatment weighting (IPTW) was used to confirm the analysis. For each patient, propensity score was calculated with logistic regression model using baseline characteristics, including age, sex, etiologies, cirrhosis, liver function, portal vein thrombosis, metastasis, AFP $>400$, sorafenib experienced, and ECOG, tumor burden as well. Generalized estimating equation was used to compare efficacy between groups.

Student's t-test was used to compare continuous variables and Chi-square test or Fisher's exact test to categorical variables between groups. Coxregression analysis was used to determine risk for 
disease progression and mortality and the Log-rank test to compare Kaplan-Meier curves. Propensityscore matching was done with caliper width of 0.1 . SPSS version 24.0 was used for the statistical analysis (IBM Corp. Released 2016. IBM SPSS Statistics for Windows, Version 24.0. Armonk, NY: IBM Corp.). $p<0.05$ was considered to indicate a statistically significant difference.

\section{Results}

\section{Patient characteristics}

Among 140 HCC patients, 58 patients had a combination of anti-PD-1 and sorafenib and 82 had anti-PD-1 alone, with the median duration of follow-up being 9.1 months. The mean of sorafenib dose in combination group was $351 \pm 168 \mathrm{mg}$. Nivolumab was prescribed for $123(87.9 \%)$ patients and pembrolizumab for 17 (12.1\%). Before matching, the combination group had significantly higher ALBI grade, more PVT $(74.1 \%$ vs. $46.3 \%, p<0.05$ ) and more advanced BCLC stage (stage C $94.8 \%$ vs. $81.7 \%, p<0.05)$. After matching, clinical variables were not different between groups (Table 1).

\section{Treatment response}

The combination therapy had a trend of higher CR rate ( $8.6 \%$ vs. $4.9 \%$, ns.), ORR $(22.4 \%$ vs. $19.5 \%$, ns.) and significantly higher DCR $(69.0 \%$ vs. $37.8 \%, p<0.05)$ comparing to anti-PD-1 alone. After matching, the higher DCR remained significant with combination therapy (Table 2). The change of tumor size could be evaluated in 122 patients, which showed combination therapy achieved more tumor shrinkage and better disease control (Fig. 1).

\section{Progression-free survival and overall survival}

After matching, combination therapy showed longer PFS (3.87 vs. 2.43 months, $p<0.05$ ) than anti-PD-1 alone (Fig. 2A). Combination therapy had a lower risk of disease progression (HR 0.62, [0.38-1.00]) and most of the subgroups favored combination therapy except age $<60$ years and no metastasis (Fig. 3A). Combination therapy achieved longer OS (not estimated vs. 7.17 months, $p<0.05$ ) (Fig. 2B) and lower risk of death (HR 0.46, [0.27-0.78]). Subgroup analysis showed all groups favored combination therapy (Fig. 3B). The time to response were not significantly different with combination therapy and anti-PD-1 alone (2.16 vs. 3.53 months,

Table 1 Demographic and clinical characteristics

\begin{tabular}{|c|c|c|c|c|c|c|c|c|c|c|}
\hline \multirow{2}{*}{ Characteristic } & \multicolumn{5}{|c|}{ Before matching } & \multicolumn{4}{|c|}{ After matching } & \multirow[b]{2}{*}{$p$ value } \\
\hline & \multicolumn{2}{|c|}{$\begin{array}{l}\text { Anti-PD-1 plus } \\
\text { Sorafenib }(n=58)\end{array}$} & \multicolumn{2}{|l|}{$\begin{array}{l}\text { Anti-PD-1 } \\
\text { alone } \\
(n=82)\end{array}$} & \multirow{2}{*}{$\begin{array}{l}p \\
\text { value }\end{array}$} & \multicolumn{2}{|c|}{$\begin{array}{l}\text { Anti-PD-1 plus } \\
\text { sorafenib }(n=58)\end{array}$} & \multicolumn{2}{|c|}{$\begin{array}{l}\text { Anti-PD-1 alone } \\
(n=42)\end{array}$} & \\
\hline Age (mean $\pm S D)$ & 69.1 & & $61.7 \pm 12.3$ & & & 69.1 & & 61.2 & & 0.25 \\
\hline Male & 45 & $77.6 \%$ & 64 & $78.0 \%$ & 0.95 & 45 & $77.6 \%$ & 29 & $69.0 \%$ & 0.77 \\
\hline \multicolumn{11}{|l|}{ Etiology } \\
\hline HBV & 35 & $60.3 \%$ & 53 & $64.6 \%$ & 0.61 & 35 & $60.3 \%$ & 28 & $66.7 \%$ & 0.52 \\
\hline HCV & 9 & $15.5 \%$ & 15 & $18.3 \%$ & 0.67 & 9 & $15.5 \%$ & 8 & $19.0 \%$ & 0.64 \\
\hline Alcohol & 29 & $50.0 \%$ & 36 & $43.9 \%$ & 0.48 & 29 & $50.0 \%$ & 18 & $42.9 \%$ & 0.48 \\
\hline Cirrhosis & 21 & $36.2 \%$ & 26 & $31.7 \%$ & 0.58 & 21 & $36.2 \%$ & 17 & $40.5 \%$ & 0.66 \\
\hline \multicolumn{11}{|l|}{ Child-Pugh score } \\
\hline A & 47 & $81.0 \%$ & 66 & $80.5 \%$ & 0.94 & 47 & $81.0 \%$ & 29 & $69.0 \%$ & 0.17 \\
\hline B & 11 & $19.0 \%$ & 16 & $19.5 \%$ & & 11 & $19.0 \%$ & 13 & $31.0 \%$ & \\
\hline \multicolumn{11}{|l|}{ ALBI grade } \\
\hline Grade 1 & 10 & $17.2 \%$ & 31 & $37.8 \%$ & $<0.05$ & 10 & $17.2 \%$ & 12 & $28.6 \%$ & 0.34 \\
\hline Grade 2 & 41 & $70.7 \%$ & 47 & $57.3 \%$ & & 41 & $70.7 \%$ & 27 & $64.3 \%$ & \\
\hline Grade 3 & 7 & $12.1 \%$ & 4 & $4.9 \%$ & & 7 & $12.1 \%$ & 3 & $7.1 \%$ & \\
\hline \multicolumn{11}{|l|}{ BCLC stage } \\
\hline B & 3 & $5.2 \%$ & 15 & $18.3 \%$ & $<0.05$ & 3 & $5.20 \%$ & 3 & $7.1 \%$ & 0.68 \\
\hline C & 55 & $94.8 \%$ & 67 & $81.7 \%$ & & 55 & $94.8 \%$ & 39 & $92.9 \%$ & \\
\hline PVT & 43 & $74.1 \%$ & 38 & $46.3 \%$ & $<0.05$ & 43 & $74.1 \%$ & 29 & $69.0 \%$ & 0.58 \\
\hline Metastasis & 30 & $51.7 \%$ & 49 & $59.8 \%$ & 0.35 & 30 & $51.7 \%$ & 26 & $61.9 \%$ & 0.31 \\
\hline AFP $>400(\mathrm{ng} / \mathrm{mL})$ & 34 & $58.6 \%$ & 43 & $52.4 \%$ & 0.47 & 34 & $58.6 \%$ & 29 & $69.0 \%$ & 0.29 \\
\hline Sorafenib experienced & 37 & $63.8 \%$ & 49 & $59.8 \%$ & 0.63 & 37 & $63.8 \%$ & 28 & $66.7 \%$ & 0.31 \\
\hline
\end{tabular}

PVT portal vein thrombosis 
Table 2 Treatment response

\begin{tabular}{clllll}
\hline Before matching & $\begin{array}{l}\text { Anti-PD-1 plus } \\
\text { sorafenib } \\
\text { ( } \boldsymbol{n = 5 8 )}\end{array}$ & $\begin{array}{l}\text { Anti-PD-1 } \\
\text { alone }(\boldsymbol{n}=\mathbf{8 2})\end{array}$ & $p$ value \\
\hline ORR & 13 & $22.4 \%$ & 16 & $19.5 \%$ & 0.68 \\
DCR & 40 & $69.0 \%$ & 31 & $37.8 \%$ & $<0.05$ \\
CR & 5 & $8.6 \%$ & 4 & $4.9 \%$ & \\
PR & 8 & $13.8 \%$ & 12 & $14.6 \%$ & \\
SD & 27 & $46.6 \%$ & 15 & $18.3 \%$ & \\
After matching & Anti-PD-1 plus & Anti-PD-1 & & $p$ value \\
& sorafenib $(n=58)$ & alone $(n=42)$ & & \\
ORR & 13 & $22.4 \%$ & 9 & $21.4 \%$ & 0.91 \\
DCR & 40 & $69.0 \%$ & 14 & $33.3 \%$ & $<0.05$ \\
CR & 5 & $8.6 \%$ & 3 & $7.1 \%$ & \\
PR & 8 & $13.8 \%$ & 6 & $14.3 \%$ & \\
SD & 27 & $46.6 \%$ & 5 & $11.9 \%$ & \\
\hline
\end{tabular}

$C R$ complete response, $P R$ partial response, $S D$ stable disease, $P D$ progressive disease, $O R R$ objective response rate, $D C R$ disease-control rate

$p=0.20$ ). The duration of response was not different neither ( 8.89 vs. 8.05 months, $p=0.45)$. After IPTW, the combination group still demonstrated decreasing risk of disease progression (HR $0.18, p$ value $<0.001$ ), and death $($ HR $0.42, p$ value $=0.03)$.

\section{Early AFP response associated with image response}

The early decline of AFP level was strongly associated with the subsequent image response, which could be observed at days14. Patients with AFP level more than 10 $(\mathrm{ng} / \mathrm{mL})$ and declined more than $10 \%$ from baseline had higher ORR ( $46 \%$ vs. $10 \%, p<0.001)$ and CR rate $(25 \%$ vs. $3 \%, p<0.001)$. At day 28, the more AFP declined ( $>10$, $>50$ and $>66 \%$ ), the higher ORR (70, 80 and $92 \%)$ and CR rate (30, 35 and 58\%) achieved (Fig. 4).

\section{Organ-specific tumor response}

The tumor response among different metastatic sites was assessed. The ORR was $19.4 \%$ in the liver $(n=129), 33.3 \%$ in lung $(n=15), 25.0 \%$ in lymph node $(n=7), 40.0 \%$ in peritoneum $(n=5), 0 \%$ in bone metastasis $(n=5)$ and $50 \%$ in adrenal metastasis $(n=4)$. Tumor thrombosis was regressed in $7.4 \%$ of cases $(n=81)$, including one CR and 2 PR in patients with PVT, 2 PR with inferior vena cava thrombosis and one $\mathrm{CR}$ with superior mesenteric vein thrombosis (Table 3).

\section{Toxicity}

Combination therapy had more grade $3 / 4 \mathrm{AE}$ without statistically significant $(10.3 \%$ vs. $7.1 \%, p=0.73)$ (Supplemental Table 1). Generally, the incidence of grade $3 / 4$ $\mathrm{AE}$ was less than $5 \%$ in each site. Both groups had hepatitis, pneumonitis and skin toxicities. Especially, there was one sick sinus syndrome developed with combination therapy, which has been published as the first case report [14].

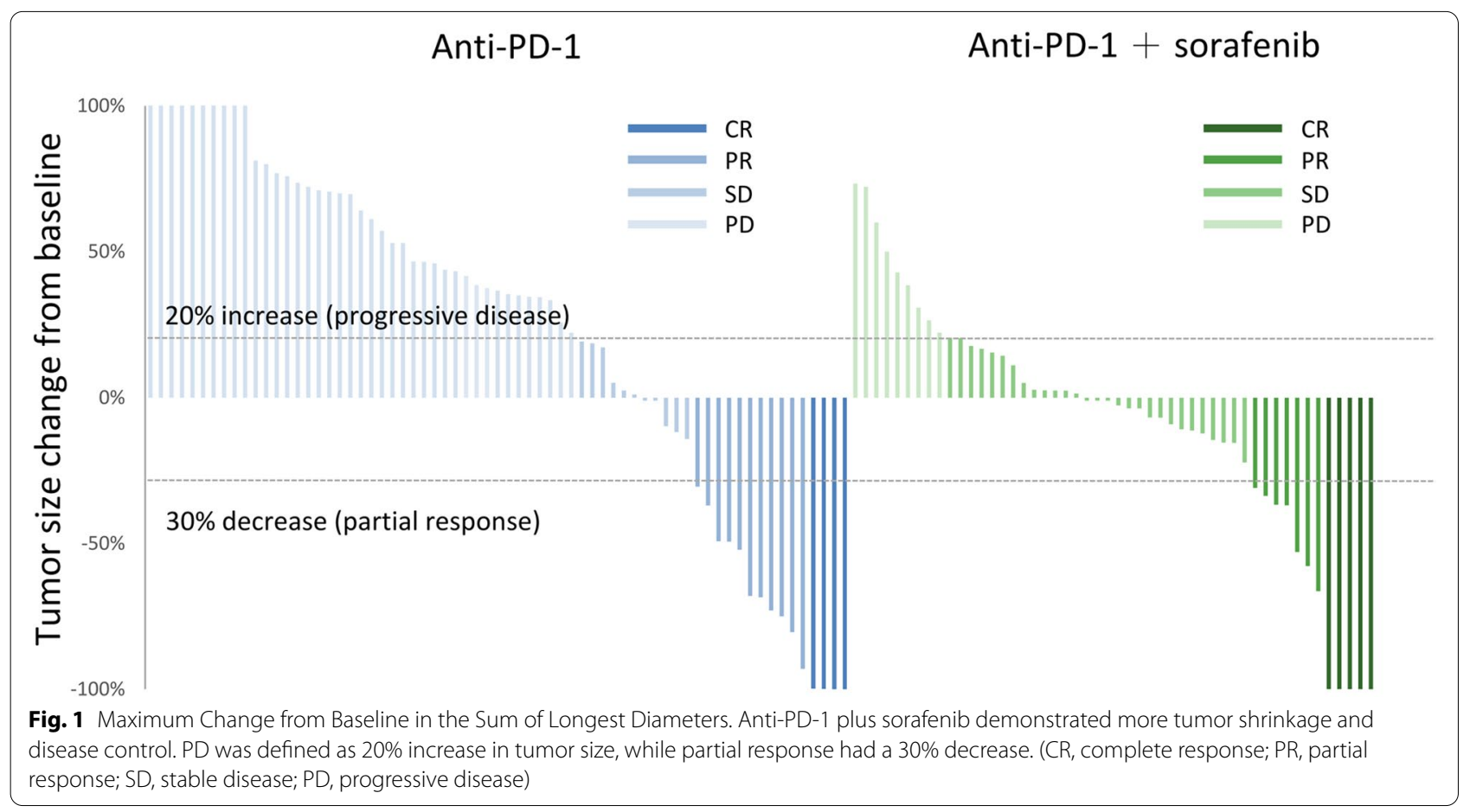



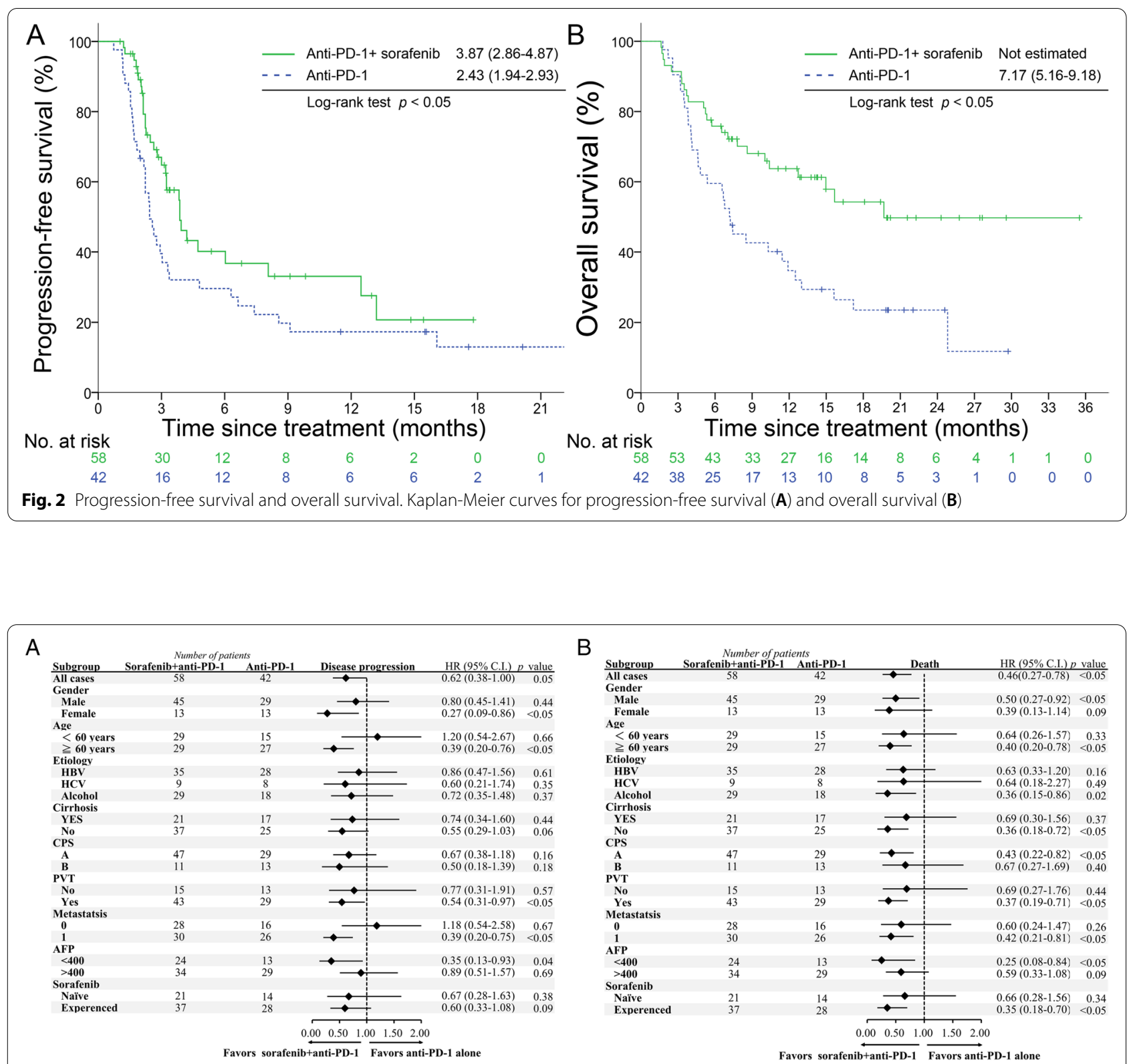

Fig. 3 Subgroup analysis in the matched cohort. Subgroup analysis for disease progression (A) or death (B). PVT denotes portal vein thrombosis

\section{Discussion}

The major findings of this study included (1) the combination of anti-PD-1 and sorafenib demonstrated better tumor control, longer PFS and OS comparing with anti-PD-1 alone, (2) combination therapy did not increase grade $3 / 4$ toxicities significantly, and (3) deeper AFP response was a surrogate marker for deeper image response.

In recent years, anti-PD-1 has become a breakthrough treatment for advanced HCC. Comparing with sorafenib, nivolumab demonstrated a higher ORR ( $15 \%$ vs. $7 \%)$, but similar DCR ( $55 \%$ vs. $58 \%$ ), PFS (3.7 vs. 3.8 months) and
OS (16.4 vs. 14.7 months) [3]. Therefore, anti-PD-1 alone failed to prove its superiority to sorafenib in the first-line treatment. In the absence of biomarker, combination of anti-PD-1 and other anti-tumor agents becomes a feasible treatment strategy to achieve better efficacy.

In this study, we found the addition of sorafenib to antiPD-1 greatly increased DCR from 33 to $69 \%$. In addition, the degree of deterioration with combination therapy was mild comparing with anti-PD-1 alone in the waterfall plot. Such efficacy translated into a lower risk of disease progression (HR 0.62) and death (HR 0.46). From past research, including SHARP and Asian-Pacific study, 


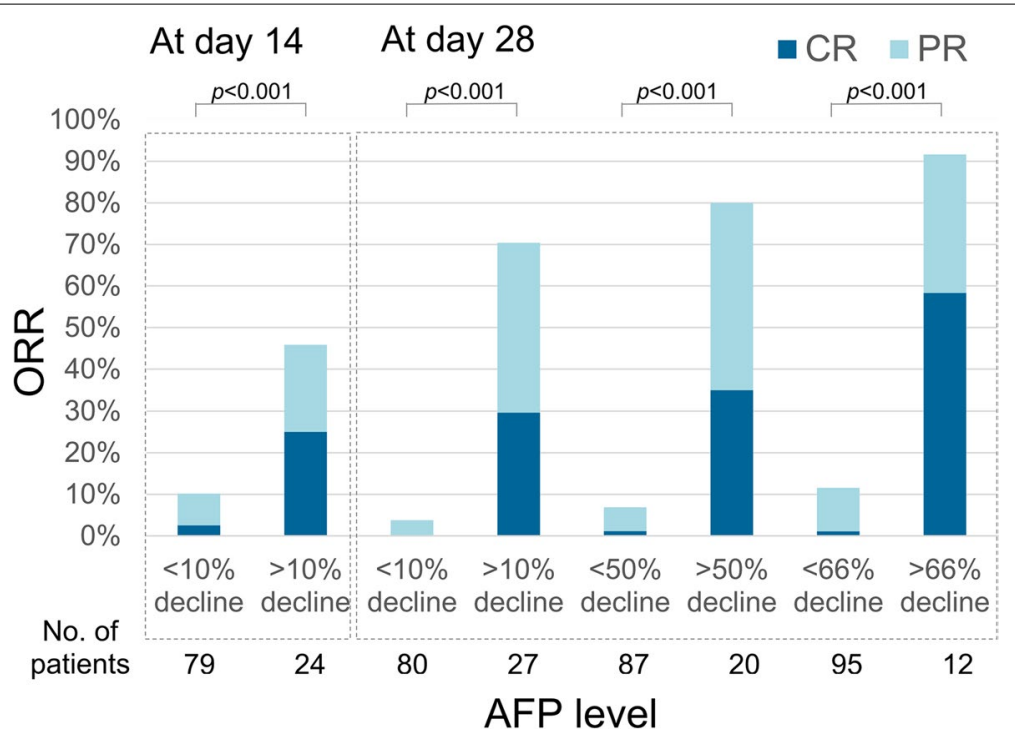

Fig. 4 The association between AFP response and image response. AFP response was strongly associated with the subsequent image response, which could be observed as early as 14 days. The more decline of AFP level, the higher ORR and CR rate. (ORR, objective response rate; CR, complete response; PR, partial response)

Table 3 Organ-specific tumor response $(n=140)$

\begin{tabular}{|c|c|c|c|c|c|c|c|c|}
\hline Sites & CR & & PR & & SD & & ORR & DCR \\
\hline Liver $(n=129)$ & 9 & $7.0 \%$ & 16 & $12.4 \%$ & 40 & $31.0 \%$ & $19.4 \%$ & $50.4 \%$ \\
\hline Thrombosis $(n=81)$ & 2 & $2.5 \%$ & 4 & $4.9 \%$ & 25 & $30.9 \%$ & $7.4 \%$ & $38.3 \%$ \\
\hline Lung $(n=15)$ & 2 & $13.3 \%$ & 3 & $20.0 \%$ & 3 & $20.0 \%$ & $33.3 \%$ & $53.3 \%$ \\
\hline Lymph node $(n=7)$ & 0 & $0.0 \%$ & 1 & $14.3 \%$ & 1 & $14.3 \%$ & $14.3 \%$ & $28.6 \%$ \\
\hline Peritoneum $(n=5)$ & 1 & $20.0 \%$ & 1 & $20.0 \%$ & 0 & $0.0 \%$ & $40.0 \%$ & $40.0 \%$ \\
\hline Bone $(n=5)$ & 0 & $0.0 \%$ & 0 & $0.0 \%$ & 1 & $20.0 \%$ & $0.0 \%$ & $20.0 \%$ \\
\hline Adrenal gland $(n=4)$ & 0 & $0.0 \%$ & 2 & $50.0 \%$ & 0 & $0.0 \%$ & $50.0 \%$ & $50.0 \%$ \\
\hline
\end{tabular}

$C R$ complete response, $P R$ partial response, $S D$ stable disease, $P D$ progressive disease, $O R R$ objective response rate, $D C R$ disease control rate

high DCR of sorafenib lowered risk of progression (HR 0.58 and 0.57 ) and death (HR 0.69 and 0.68) comparing with placebo $[7,15]$. These data suggested that sorafenib retains its anti-tumor properties when combined with anti-PD-1.

At present, a convenient and reliable biomarker for the prediction of anti-PD-1 response in HCC is still lacking. However, some evidence showed that the change of AFP level was corelated to anti-PD-1 response. We previously found that patients with AFP $>10 \mathrm{ng} / \mathrm{dL}$ before treatment and declined $>10 \%$ within 4 weeks could predict image response [16]. Shao et al. used a $20 \%$ decline as the cutoff level of the AFP response [17]. In this study, we found that the response of AFP on day 14 can early predict the subsequent image response. This may suggest that antiPD-1 can exert its anti-tumor effect at such an early stage. Furthermore, we found that the more AFP level declined, the deeper tumor response. Patients with AFP declined $>66 \%$ on day 28 , the CR rate could even reach $58 \%$. Conversely, when AFP declined less than $10 \%$, the ORR was only $3 \%$. These further addressed the important negative predictive value of AFP response. Taken together, the change of AFP value is parallel to that of the image response.

Grade 3/4 irAE was about 25\% with nivolumab and pembrolizumab alone, but it rose to $29-53 \%$ with the combination of nivolumab and ipilimumab [18], 56\% with atezolizumab and bevacizumab [6], and 73\% with pembrolizumab and lenvatinib [19]. In our study, grade $3 / 4$ irAE was only $10.3 \%$ with the combination of antiPD-1 and sorafenib. How to strike a balance between efficacy and toxicity remained an important issue at present. Furthermore, the median time to onset of high grade irAE was 20 days in this study, which is compatible with a previous report that showed fatal toxic effects typically occurred early ( 14.5 days) after 
immunotherapy initiation [20]. Therefore, caution is advised during this period when the risk of fetal irAE is high but the efficacy can't be estimated yet.

Regarding organ-specific tumor response, lung and peritoneal metastasis have the highest response rates, followed by liver metastasis in a melanoma study [21]. In HCC, Lu et al. published a similar result, which showed the highest response rates were the lungs (41.2\%) and intra-abdomen (38.9\%), followed by the lymph node (26.3\%) and liver (22.4\%) (28). Our study showed that the metastatic organ of the lungs (33.4\%), peritoneum $(40.0 \%)$ and adrenal glands $(50.0 \%)$ had high response rate, followed by the liver (19.4\%) and lymph node (14.3\%). Conversely, the response of bone metastasis and tumor thrombosis was quite low $(<10 \%)$. Such consistency implies that the tumor microenvironment of various organs may potentially influence the therapeutic effect.

This study has several limitations. First, this is a retrospective study, so selection bias is inevitable. With the use of propensity-score matching analysis, we minimized the bias between groups. Second, the mean of sorafenib starting doses in this study was about half of the recommended dose, which may underestimate the efficacy of combination therapy. However, the optimal escalation strategy of sorafenib is controversial [22]. The adequate dose of multi-kinase inhibitors to combine with anti-PD-1 is also unclear. Therefore, this study provided an important reference for such combination. Finally, to avoid recall bias for low grade AE, this article only analyzed grade $3 / 4 \mathrm{AE}$.

\section{Conclusions}

This propensity-score matching study showed that the combination of anti-PD-1 and sorafenib had better tumor control and prolonged PFS and OS with tolerated toxic profile in advanced HCC. However, a welldesigned prospective randomized study is needed to confirm this finding.

\section{Abbreviations}

VEGF: Vascular endothelial growth factor; HCC: Hepatocellular carcinoma; MKI: Multiple-kinase inhibitors; BCLC: Barcelona clinic liver cancer; PFS: Progressionfree survival; ToR: Time to response; DoR: Duration of response; OS: Overall survival; PVT: Portal vein thrombosis.

\section{Supplementary Information}

The online version contains supplementary material available at https://doi. org/10.1186/s12885-022-09173-4.

Additional file 1 : Supplemental Table 1. Grade 3/4 adverse event. Additional file 2 .

\section{Acknowledgements}

The author wishes to acknowledge the support by Ministry of Health and Welfare and the Center of Excellence for Cancer Research (MOHW110-TDUB-211-144019), Taipei Veterans General Hospital (V107B-036).

\section{Authors' contributions}

Conceived and designed the experiments: S-CC, YC. Performed the experiments: S-CC, Y-HH. Analyzed the data: S-CC, M-HC, R-CL. Contributed reagents/materials/analysis tools: $\mathrm{Y}-\mathrm{HH}, \mathrm{M}-\mathrm{HC}, \mathrm{Y}-\mathrm{PH}, \mathrm{Y}-\mathrm{YS}$. Contributed to the writing of the manuscript: S-CC, Y-PH, Y-PH. The author(s) read and approved the final manuscript.

\section{Funding}

Ministry of Health and Welfare and the Center of Excellence for Cancer Research (MOHW1 10-TDU-B-211-144019), Taipei Veterans General Hospital (V107B-036 to S-CC)

Availability of data and materials

Data available on request from the authors.

\section{Declarations}

\section{Ethics approval and consent to participate}

This study has been approved by the institutional review board of Taipei Veterans General Hospital, which is the appropriate regulatory agency to review research on both adults and children. All methods were carried out in accordance with relevant guidelines and regulations.

The subject informed consent form is waived by the institutional review board of Taipei Veterans General Hospital, and study-related information could be not informed the subjects.

\section{Consent for publication}

NA.

\section{Competing interests}

The authors declare no conflict of interest.

\section{Author details}

${ }^{1}$ Institute of Clinical Medicine, National Yang Ming Chiao Tung University, Taipei, Taiwan. ${ }^{2}$ Faculty of Medicine, National Yang Ming Chiao Tung University, Taipei, Taiwan. ${ }^{3}$ Division of Medical Oncology, Center for Immuno-oncology, Department of Oncology, Taipei Veterans General Hospital, No. 201, Sec. 2, Shipai Road, Taiwan 11217 Taipei, Taiwan. ${ }^{4}$ Division of Gastroenterology and Hepatology, Department of Medicine, Taipei Veterans General Hospital, Taipei, Taiwan. ${ }^{5}$ Department of Radiology, Taipei Veterans General Hospital, Taipei, Taiwan. ${ }^{6}$ Department of Oncology, National Taiwan University Hospital, Taipei, Taiwan. ${ }^{7}$ Graduate Institute of Oncology, National Taiwan University College of Medicine, Taipei, Taiwan. ${ }^{8}$ Department of Medical Oncology, National Taiwan University Cancer Center, Taipei, Taiwan.

Received: 27 July 2021 Accepted: 24 December 2021 Published online: 11 January 2022

References

1. The leading causes of death in Taiwan. 2018. Retrieved March 1, 2020, from https://www.mohw.gov.tw/cp-16-48057-1.html

2. International Agency for Research on Cancer, World Health Organization. Cancer today. Retrieved March 1, 2020, from https://gco.iarc.fr/today/ home.

3. Yau T, Park JW, Finn RS, Cheng AL, Mathurin P, Edeline J, et al. CheckMate 459: a randomized, multi-center phase III study of nivolumab (NIVO) vs sorafenib (SOR) as first-line (1L) treatment in patients (pts) with advanced hepatocellular carcinoma (aHCC). Ann Oncol. 2019;30:v874-5.

4. Fukumura D, Kloepper J, Amoozgar Z, Duda DG, Jain RK. Enhancing cancer immunotherapy using antiangiogenics: opportunities and challenges. Nat Rev Clin Oncol. 2018;15:325-40.

5. Lee MS, Ryoo BY, Hsu CH, Numata K, Stein S, Verret W, et al. Atezolizumab with or without bevacizumab in unresectable hepatocellular carcinoma 
(GO30140): an open-label, multicentre, phase 1b study. Lancet Oncol. 2020;21:808-20.

6. Finn RS, Qin S, Ikeda M, Galle PR, Ducreux M, Kim TY, et al. Atezolizumab plus Bevacizumab in Unresectable Hepatocellular Carcinoma. N Engl J Med. 2020;382:1894-905.

7. Llovet JM, Ricci S, Mazzaferro V, Hilgard P, Gane E, Blanc JF, et al. Sorafenib in advanced hepatocellular carcinoma. N Engl J Med. 2008;359:378-90.

8. Chen ML, Yan BS, Lu WC, Chen MH, Yu SL, Yang PC, et al. Sorafenib relieves cell-intrinsic and cell-extrinsic inhibitions of effector $T$ cells in tumor microenvironment to augment antitumor immunity. Int I Cancer. 2014;134:319-31.

9. Cao M, Xu Y, Youn Jl, Cabrera R, Zhang X, Gabrilovich D, et al. Kinase inhibitor Sorafenib modulates immunosuppressive cell populations in a murine liver cancer model. Lab Investig. 2011;91:598-608.

10. Chuang HY, Chang YF, Liu RS, Hwang JJ. Serial low doses of sorafenib enhance therapeutic efficacy of adoptive $T$ cell therapy in a murine model by improving tumor microenvironment. PLoS One. 2014:9:e109992.

11. Lu LC, Lee YH, Chang CJ, Shun CT, Fang CY, Shao YY, et al. Increased expression of programmed death-ligand 1 in infiltrating immune cells in hepatocellular carcinoma tissues after Sorafenib treatment. Liver Cancer. 2019:8:110-20

12. Wang Y, Li H, Liang Q, Liu B, Mei X, Ma Y. Combinatorial immunotherapy of sorafenib and blockade of programmed death-ligand 1 induces effective natural killer cell responses against hepatocellular carcinoma. Tumour Biol. 2015;36:1561-6.

13. Chen SC, Chao Y, Yang MH. Complete response to the combination of pembrolizumab and sorafenib for metastatic hepatocellular carcinoma: a case report. Am J Gastroenterol. 2017;112:659-60.

14. Hsu CY, Su YW, Chen SC. Sick sinus syndrome associated with anti-programmed cell death-1. J Immunother Cancer. 2018:6:72.

15. Cheng AL, Kang YK, Chen Z, Tsao CJ, Qin S, Kim JS, et al. Efficacy and safety of sorafenib in patients in the Asia-Pacific region with advanced hepatocellular carcinoma: a phase III randomised, double-blind, placebocontrolled trial. Lancet Oncol. 2009;10:25-34

16. Lee P-C, Chao Y, Chen M-H, Lan K-H, Lee C-J, Lee IC, et al. Predictors of response and survival in immune checkpoint inhibitor-treated unresectable hepatocellular carcinoma. Cancers. 2020;12:182.

17. Shao YY, Liu TH, Hsu C, Lu LC, Shen YC, Lin ZZ, et al. Early alphafetoprotein response associated with treatment efficacy of immune checkpoint inhibitors for advanced hepatocellular carcinoma. Liver Int. 2019:39:2184-9.

18. Yau T, Kang Y-K, Kim T-Y, El-Khoueiry AB, Santoro A, Sangro B, et al. Nivolumab (NIVO) + ipilimumab (IPI) combination therapy in patients (pts) with advanced hepatocellular carcinoma (aHCC): results from CheckMate 040. J Clin Oncol. 2019;37:4012.

19. Llovet J, Shepard KV, Finn RS, Ikeda M, Sung M, Baron AD, et al. A phase Ib trial of lenvatinib (LEN) plus pembrolizumab (PEMBRO) in unresectable hepatocellular carcinoma (uHCC): updated results. Ann Oncol. 2019;30:v286-7.

20. Wang DY, Salem JE, Cohen JV, Chandra S, Menzer C, Ye F, et al. Fatal toxic effects associated with immune checkpoint inhibitors: a systematic review and meta-analysis. JAMA Oncol. 2018;4:1721-8.

21. Khoja L, Kibiro M, Metser U, Gedye C, Hogg D, Butler MO, et al. Patterns of response to anti-PD-1 treatment: an exploratory comparison of four radiological response criteria and associations with overall survival in metastatic melanoma patients. Br J Cancer. 2016;115:1186-92.

22. Tak KY, Nam HC, Choi JY, Yoon SK, Kim CW, Kim HY, et al. Effectiveness of sorafenib dose modifications on treatment outcome of hepatocellular carcinoma: analysis in real-life settings. Int J Cancer. 2020;147:1970-8.

\section{Publisher's Note}

Springer Nature remains neutral with regard to jurisdictional claims in published maps and institutional affiliations.
Ready to submit your research? Choose BMC and benefit from:

- fast, convenient online submission

- thorough peer review by experienced researchers in your field

- rapid publication on acceptance

- support for research data, including large and complex data types

- gold Open Access which fosters wider collaboration and increased citations

- maximum visibility for your research: over $100 \mathrm{M}$ website views per year

At BMC, research is always in progress.

Learn more biomedcentral.com/submissions 